BMJ Open

Sport \&

Exercise

Medicine

\title{
Physiotherapy and physical activity: a cross-sectional survey exploring physical activity promotion, knowledge of physical activity guidelines and the physical activity habits of UK physiotherapists
}

Anna Lowe, Chris Littlewood, ${ }^{1}$ Sionnadh McLean, ${ }^{2}$ Karen Kilner ${ }^{2}$

To cite: Lowe A, Littlewood C, McLean S, et al. Physiotherapy and physical activity: a crosssectional survey exploring physical activity promotion, knowledge of physical activity guidelines and the physical activity habits of UK physiotherapists. BMJ Open Sport Exerc Med 2017;3: e000290. doi:10.1136/ bmjsem-2017-000290

Accepted 1 September 2017

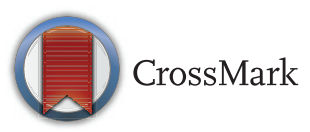

${ }^{1}$ Arthritis Research UK Primary Care Centre, Research Institute for Primary Care and Health Sciences and Keele Clinical Trials Unit, David Weatherall Building, Keele University, Staffordshire, UK

${ }^{2}$ Centre for Health and Social Care Research, Sheffield Hallam University, Sheffield, UK

\section{Correspondence to} Anna Lowe, Centre for Health and Social Care Research, Sheffield Hallam University, Sheffield, UK; a.lowe@shu. ac.uk

\section{ABSTRACT}

Objective Physical inactivity is a public health priority and embedding promotion of physical activity (PA) within healthcare systems is an important lever for change. Many factors influence PA promotion in routine healthcare practice; these include the PA habits of healthcare professionals and also their knowledge of the PA guidelines. Little is known about the extent to which PA is currently promoted in physiotherapy practice or the factors that influence it.

Methods Following ethical approval, a cross-sectional survey of UK physiotherapists was conducted. Findings were analysed and reported in accordance with STROBE (STrengthening the Reporting of OBservational studies in Epidemiology) guidelines. Results There were 522 respondents, 514 of whom were physiotherapists. Seventy-seven per cent of respondents routinely discussed PA with patients and $68 \%$ routinely delivered brief interventions.

Assessment of PA status was not routine practice, neither was signposting to further sources of PA support. Only $16 \%$ of respondents correctly answered questions about the content of the PA guidelines. Only $38 \%$ of respondents met current PA recommendations. Clinicians' PA levels were not associated with PA promotion activity.

Conclusion Despite the promising finding that some form of PA promotion is integrated into most respondents' practice, we report a poor understanding of brief interventions and poor knowledge of the PA guidelines. Additionally, the majority of respondents were not sufficiently active to meet current PA recommendations.

\section{BACKGROUND}

Physical inactivity (PI), defined as achieving less than 30 min physical activity (PA) per week, ${ }^{1}$ has a significant impact on morbidity and mortality which leads to economic burden on healthcare systems and wider society. $^{2}{ }^{3}$ Inactive people

\section{What are the new findings?}

Discussions about physical activity (PA) were integrated into the majority of physiotherapy contacts.

- Brief interventions may not be carried out optimally.

- PA status of patients was not routinely assessed.

- Although $60 \%$ of physiotherapists knew that 150 min of moderate PA per week is recommended, only $16 \%$ of physiotherapists successfully answered all three questions relating to the PA guidelines.

- Physiotherapists did not routinely signpost to further sources of PA support.

- The majority of physiotherapists were not sufficiently active to meet the current PA guidelines for adults.

\section{How might it impact on clinical practice in} the near future?

- To maximise the potential impact of physiotherapy on physical inactivity we recommend.

- Further efforts to disseminate the current PA guidelines.

- Targeted knowledge translation of brief interventions for PA in a physiotherapy context.

spend 38\% more days in hospital and use significantly more healthcare resources than active people. ${ }^{4}$ Accordingly, there is national and international guidance on how PA can be promoted..$^{5-7}$ 
Many factors influence PA at population level and meaningful change requires sustained efforts across multiple systems. ${ }^{8}$ Healthcare is one such system and integrating PA promotion into healthcare is one of the seven 'best investments' for reducing PI. ${ }^{9}$

Every healthcare contact is an opportunity to positively influence a patient's health and this is often done pragmatically through brief interventions. Brief interventions for PA can be delivered in routine healthcare consultations; they have the potential to reach a large proportion of the adult population and have been shown to be cost-effective. ${ }^{10}{ }^{11}$ Clinical guidance recommends the use of brief interventions in routine clinical contacts and this forms part of a wider Making Every Contact Count approach which is now embedded within National Health Service (NHS) delivery in the $\mathrm{UK}^{12} 13$

Physiotherapists are well placed to promote PA. There are over 51000 physiotherapists registered in the UK working across health and social care, often supporting people with long-term conditions. ${ }^{14}$ In 2015-2016, there were over 5 million physiotherapy outpatient contacts ${ }^{15}$; a large proportion of patients accessing outpatient physiotherapy services are either overweight or obese, have multiple comorbid health conditions and are physically inactive. ${ }^{16}$

Little is known about the extent to which PA promotion is currently integrated into physiotherapy practice. Several international studies suggest that levels of PA promotion in physiotherapy practice are low. ${ }^{17-21}$ One study from Ireland presented more positive findings ${ }^{22}$ and we identified no studies that explore the extent of PA promtion in a UK setting in a 2016 scoping review. $^{23}$

Delivering brief interventions for PA requires healthcare professionals to have knowledge of PA guidelines. Specifically, healthcare professionals must ascertain whether a patient is in a risk category and know how to make evidence-based recommendations. The first UKwide PA guidelines were published in $2011^{5}$; these were updated and formatted into an infographic in $2015 .^{24}$

Previous studies suggest PA guidelines are insufficiently taught in undergraduate medical curricula and there is lack of knowledge of them among final year medical students. ${ }^{25-27} \mathrm{~A}$ survey of physiotherapists in Ireland reported that only $51 \%$ of participants were able to accurately state the current PA guidelines. ${ }^{17}$ No studies have been identified that explore knowledge of PA guidelines among UK physiotherapists.

A healthy and productive NHS workforce is critical to the sustainability of the NHS and PA is an important means of improving workforce health. ${ }^{28}$ NHS organisations are encouraged to support employees to be more physically active 29 $^{30}$ yet little is known about the PA habits of the physiotherapy workforce. In addition, PA habits are a consistent and independent correlate of PA promotion in other healthcare professions. ${ }^{31}$ There is preliminary evidence that this relationship also extends to physiotherapists, ${ }^{18} 21{ }^{32}$ but this has not been explored in the UK.

Hence, the aim of this study was to explore PA promotion in routine physiotherapy practice in the UK. Specific objectives were to:

1. Understand the frequency with which physiotherapists:

i. initiate conversations about PA;

ii. formally assess PA status;

iii. deliver brief interventions for PA;

iv. signpost patients to other PA services.

2. Assess physiotherapists' knowledge of the PA guidelines.

3. Measure the PA habits of physiotherapists and evaluate whether this is associated with PA promotion in clinical practice.

\section{METHODS}

Ethics

Ethical approval was granted from the Health \& Wellbeing Faculty Ethics Committee at Sheffield Hallam University (reference 2015-16/HWB-HSC-21). The approval was for a broader survey of allied health professionals' engagement with public health practice. The specific PA questions were included with prior consent of all parties with the intention of separate analysis and publication.

\section{Design}

A cross-sectional survey was undertaken.

\section{Survey tool}

A survey tool was developed using Survey Monkey software. Following a pilot (48 physiotherapists), a small number of minor changes were made to the wording of questions. All questions were closed with finite answer options; this was agreed in view of the anticipated volume of responses (survey questions can be seen in online supplementary file 1). The questions were designed specifically for this survey with the exception of measurement of clinicians' PA habits; a validated, single-item question was used to gather this information. ${ }^{33}$ The full survey was approved by representatives from Public Health England and the Chartered Society of Physiotherapy.

\section{Procedure}

The survey was live in May 2016 and was available for 3 weeks; this was determined by the need to avoid periods of political sensitivity. The survey was promoted widely on social media and through the Chartered Society of Physiotherapy's member networks. To meet the eligibility criteria, respondents 
Table 1 Frequency with which respondents act on physical inactivity when there is a clear indication to do so

\begin{tabular}{|c|c|c|c|c|c|c|c|c|}
\hline & \multicolumn{2}{|l|}{ Never } & \multicolumn{2}{|c|}{ Sometimes } & \multicolumn{2}{|l|}{ Usually } & \multicolumn{2}{|l|}{ Always } \\
\hline & Count & $\%$ & Count & $\%$ & Count & $\%$ & Count & $\%$ \\
\hline Do you initiate conversations about PA? & 0 & 0 & 11 & 2.4 & 95 & 21 & 347 & 76.6 \\
\hline Do you assess PA status? & 96 & 21.2 & 63 & 14 & 113 & 24.9 & 181 & 40 \\
\hline Do you deliver brief interventions for PA? & 13 & 2.9 & 26 & 5.7 & 106 & 23.4 & 308 & 68 \\
\hline Do you signpost to other PA support? & 18 & 4 & 83 & 18.3 & 152 & 33.6 & 200 & 44.2 \\
\hline
\end{tabular}

PA, physical activity.

were asked to confirm that they were physiotherapists in the UK and had current patient contact.

\section{Analysis}

All returned surveys were included in the analysis regardless of missing data; consequently, the number of total responses for each survey item is varied. A decision was made to include the pilot data in analysis as only minor changes had been made to the survey following the pilot. All responses were exported into IBM SPSS V.24 and analysed using descriptive statistics. Associations between variables were assessed using $\chi^{2}$ test.

Reporting is in line with the STROBE (STrengthening the Reporting of OBservational studies in Epidemiology) statement for cross-sectional studies (Supplementary file 3). ${ }^{34}$

\section{FINDINGS}

\section{Participant characteristics}

There were 522 responses to the survey; the physiotherapy population is estimated to be $51000,{ }^{14}$ the sample is therefore approximately $1 \%$ of the estimated population.

Eighty-nine per cent $(n=463)$ of the sample were qualified physiotherapists, $10 \%(\mathrm{n}=51)$ were student physiotherapists and $1.5 \% \quad(n=8)$ were support workers. Ninety-three per cent were from England and all four nations were represented (Scotland 3\%, Wales $3 \%$ and Northern Ireland $1 \%$ ).

Respondents reported a range of years of experience (see figure 1). The majority of respondents worked in the NHS (92\%) and respondents worked across a range of settings (see figure 2).

Only findings from qualified physiotherapists and student physiotherapists are reported hereafter, they are reported together as 'physiotherapists' in line with the aims of the study. Full results are available in online supplementary file 2.

\section{Current practice}

Participants were asked to estimate the frequency with which they carried out a number of specific actions related to PA promotion in predefined categories in line with previous similar cross-sectional surveys. ${ }^{22}$ The questions were worded such that it was clear that the question related to situations in which there was a clear indication to promote PA. Findings are presented in table 1 .

\section{Knowledge of PA guidelines}

Eighty-eight per cent of respondents $(n=382)$ reported that they were aware of the existence of PA guidelines. Knowledge of three specific aspects of the recommendations is detailed in table 2. Only $16 \%(n=83)$ of respondents answered all three questions correctly.

Table 2 Table showing correct answers to PA guideline questions

\begin{tabular}{lll} 
PAG questions & $\begin{array}{l}\text { Number of correct } \\
\text { responses }\end{array}$ & $\begin{array}{l}\text { Percentage of respondents who } \\
\text { answered correctly (\%) }\end{array}$ \\
\hline $\begin{array}{l}\text { Q: How many minutes of moderate intensity physical activity is } \\
\text { recommended per week for adults? }\end{array}$ & 240 & 60 \\
A: 150 & \\
$\begin{array}{l}\text { Q: How many minutes of vigorous intensity physical activity is } \\
\text { recommended per week for adults? }\end{array}$ & 122 & 33 \\
A: 75 & & \\
$\begin{array}{l}\text { Q: On how many days per week is it recommended that adults } \\
\text { undertake strength training? }\end{array}$ & 121 & 32 \\
A: 2 & & \\
\hline
\end{tabular}




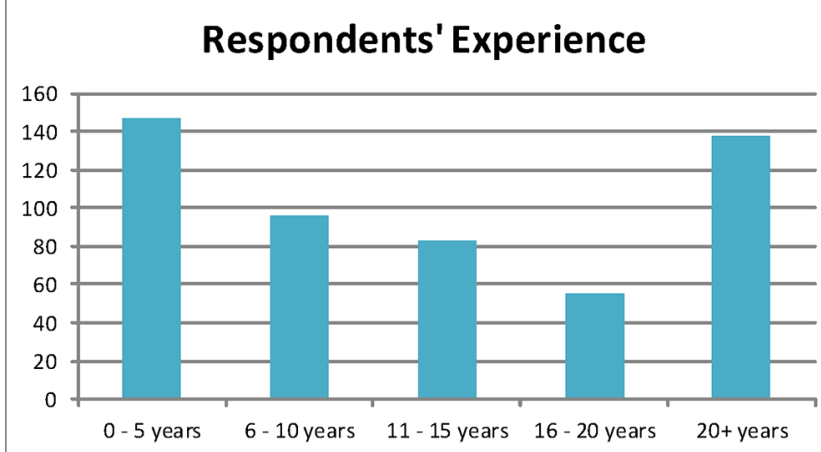

Figure 1 Years of experience of survey respondents.

\section{PA habits of physiotherapists}

The median number of sufficiently active days (ie, days on which respondents achieved at least $30 \mathrm{~min}$ of moderate $\mathrm{PA})^{33}$ was 4 . The proportion of respondents who achieved the recommended $5 \times 30 \mathrm{~min}$ of moderate intensity PA over a week was $38 \%(n=149)$.

The frequency with which respondents delivered brief interventions was not associated with years of experience $\left(\chi^{2} p=0.429\right)$ nor was it associated with physiotherapists' own PA habits $\left(\chi^{2} \mathrm{p}=0.078\right)$.

\section{DISCUSSION}

The vast majority of respondents integrated some form of discussion about PA with their patients. However, levels of PA are not routinely assessed and brief interventions are not routinely delivered. Knowledge of all three elements of the PA guidelines is poor, and therefore when brief interventions are delivered they may not be based on the best available evidence. The majority of respondents do not themselves do sufficient PA to confer optimal health benefits although this was not associated with the likelihood of them promoting $\mathrm{PA}$ in practice.

\section{How does this fit with previous research?}

There is no existing evidence on the extent to which PA promotion is integrated into physiotherapy practice in the UK. As physiotherapists in other countries provide even lower levels of PA promotion, ${ }^{17-21}$ our findings may reflect the growing awareness of PA as a major public health issue in the UK over time.

We find it encouraging that $68 \%$ of respondents report routinely delivering brief interventions for PA. This does, however, raise questions about why the other 32\% do not. Barriers to delivering brief interventions for PA in a UK physiotherapy context have not been explored; related literature suggests that barriers may include (1) lack of time, (2) lack of belief in the effectiveness of brief interventions, (3) perceived lack of knowledge, and (4) a sense that it is not acceptable to patients. ${ }^{21} 223536$
Healthcare Setting of Respondents

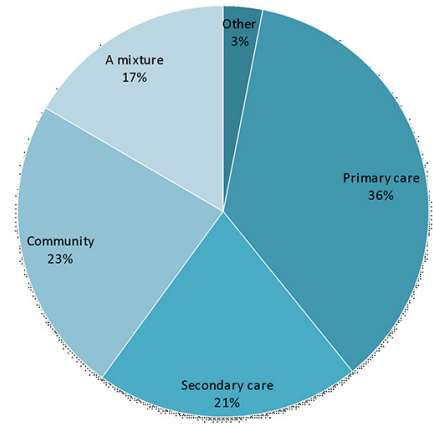

Figure 2 Healthcare setting in which respondents work.

Only $40 \%$ of respondents reported that they routinely used some form of measurement tool to assess patients' levels of PA and thus identify inactive patients, in line with earlier findings. ${ }^{17}$ Conversely, O'Donoghue et al found that $76 \%$ of physiotherapists always assessed PA levels. ${ }^{22}$ The discrepancy between these studies may relate to the definition of 'assessment'; this term could be interpreted as use of either a formal or informal approach to assessment.

Formal assessment would involve use of a measurement tool; current clinical guidance recommends the use of the General Practice Physical Activity Questionnaire to assess $\mathrm{PA}$ levels in routine practice. $^{37} 38$ However, such measures take time to complete and interpret and therefore may not be practical in a busy clinical setting.

The alternative is to use informal approaches which, although quicker, are likely to be insufficient to accurately measure PA levels and inadequate as a baseline from which to measure change. Some may argue that formal assessment is unnecessary and beyond the scope of routine practice; however, delivering brief interventions indiscriminately regardless of risk has cost implications for services that could be avoided with a more targeted approach. ${ }^{39}$

As many as $56 \%$ of respondents did not routinely direct patients to further sources of support for PA, even when there was a clear indication to do so. In other areas of health promotion, uptake of further support has been shown to be enhanced when onward referral is facilitated by the system following a brief intervention, for example, by making a forward referral at that time, rather than leaving it to patients to initiate further action themselves. ${ }^{40} 41$

Despite $88 \%$ of respondents being aware of the current Chief Medical Officers' PA guidelines, only $16 \%$ answered the three specific questions correctly. This extends evidence from other professions which highlighted a lack of curriculum content and a lack of knowledge among students. ${ }^{25-27}$ It adds weight to the recent assertion by Reid et al that that basic knowledge of the PA guidelines, and their components, remains consistently low across health professionals. ${ }^{42}$ 


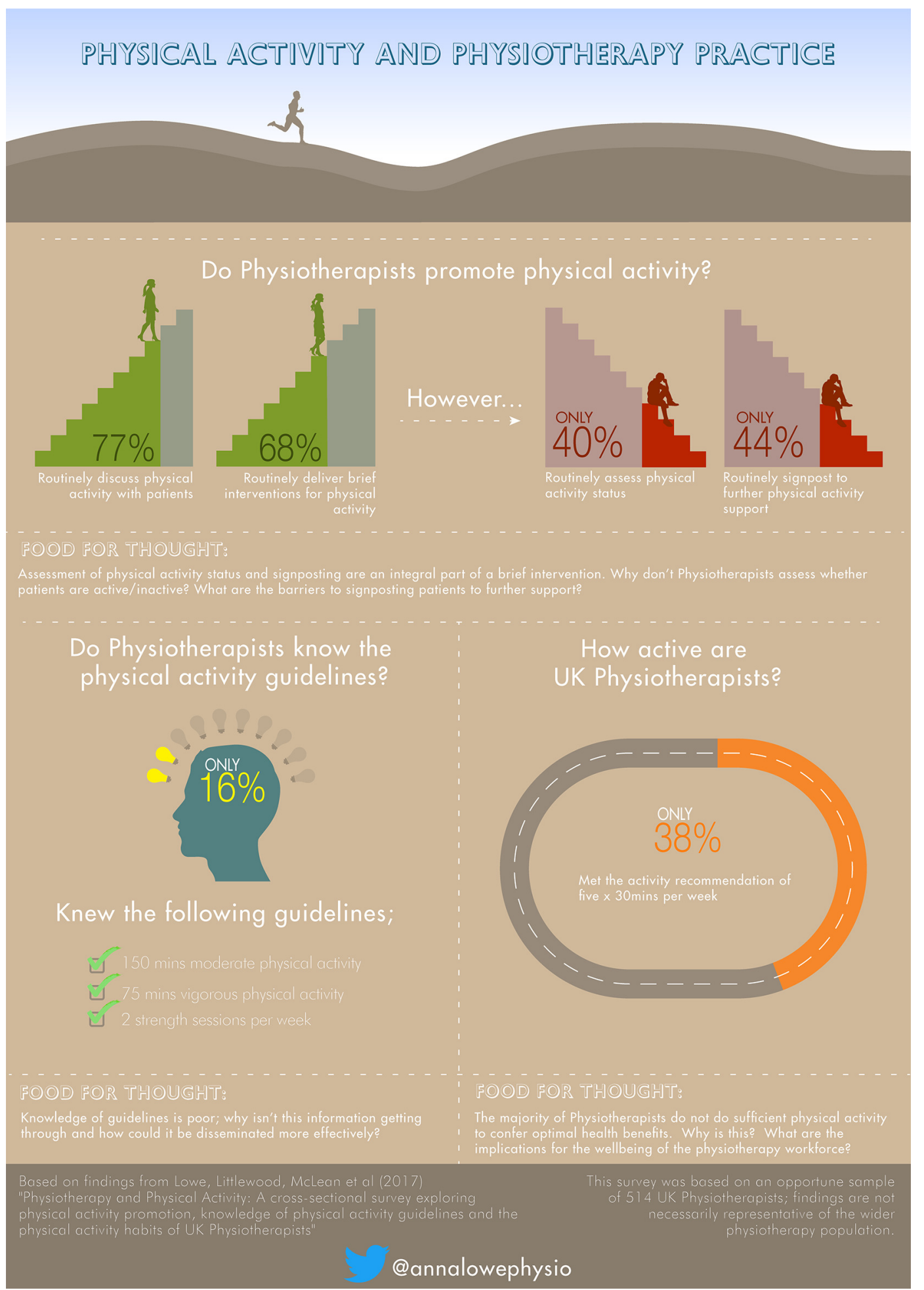

Figure 3 Physical activity and physiotherapy practice.

Only $38 \%$ of respondents achieved the recommended $5 \times 30$ min of moderate PA. This finding must be interpreted with caution due to the limitations inherent with any single-item, self-report measure. ${ }^{43} 44$ The measure used in this study excludes the incidental PA that occurs through occupation or housework for example. It therefore does not reflect the most recent iteration of the PA guidelines which promote the accumulation of $150 \mathrm{~min}$ moderate PA in bouts of 10 min or more through any means. ${ }^{5}$ In contrast to findings from other studies, physiotherapists' own PA levels were not associated with their PA promotion activity in our study. ${ }^{182132}$

\section{Suggestions for enhancing clinical practice}

Knowledge of all three elements of the PA guidelines is limited, and this raises questions about the content, 
quality and specificity of the brief interventions that are delivered in clinical practice. Additionally, assessment of PA status and signposting could be considered to be integral components of a brief intervention, yet these were delivered far less frequently. In practice, despite the number of PA measurement tools available, it is difficult to identify a tool that is sufficiently rigorous yet retains clinical utility in a physiotherapy setting. Consensus on the level of assessment of PA status that is appropriate and feasible may help improve the consistency of practice in this area. It may require a physiotherapy-led consensus statement on PA brief interventions to resonate more fully with the physiotherapy community than one led by physicians or public health experts.

The expectation that physiotherapists will signpost patients to further sources of PA support requires more investigation and mechanisms that could facilitate the sharing of PA information across sectors need to be explored.

Physiotherapists' understanding of brief interventions warrants further exploration as do the barriers to delivering them in the context of UK physiotherapy practice. In addition, further work is required to explore why the guideline specifics of training intensity and strength training are not reaching frontline clinicians. Effective dissemination of this information is required across the future and current physiotherapy workforce.

The majority of respondents to this survey were insufficiently active to gain optimum health benefits. The PA levels of the NHS workforce are an important consideration as part of a broader workforce well-being agenda. Thus, further investigation of the PA levels of the physiotherapy workforce using more robust, direct measurement techniques is warranted.

\section{Strengths and limitations}

This is the first cross-sectional survey that explores current practice in relation to PA promotion in physiotherapy practice, knowledge of PA guidelines and PA habits of physiotherapists. It has generated a preliminary picture that can inform practice developments and future research.

The UK PA guidelines contain several important messages and not all of these were tested. There are also specific PA guidelines for early years, children and older adults; only the adult guidelines were considered in this study.

The non-probability (self-selected) sampling strategy means that care must be taken when interpreting findings. The survey may have been subject to selfselection bias, with engaged physiotherapists responding more readily than those who do not have an interest in this area. This could have led to an overrepresentation of the extent to which $\mathrm{PA}$ is currently promoted for example. However, in addition to budget constraints, it would be challenging to obtain a national random sample of UK physiotherapists due to a lack of availability of essential demographic data, and thus an inability to define the population.

\section{CONCLUSION}

We identified positive findings in that most respondents integrate discussions about PA into most of their patient contacts. Further investigation is needed relating to the lack of formal assessment of PA status, relatively poor knowledge of the PA guidelines and a lack of consistent signposting to further PA support. Physiotherapists are ideally placed to contribute to the global efforts to reduce PI. However,support is required to ensure that effective and feasible PA interventions are integrated into routine care in order to maximise potential impact. To improve the reach of our study we have created a summary infographic (figure 3).

\section{Twitter @annalowephysio}

Contributors This study was led by $\mathrm{AL}$ under the supervision of $\mathrm{SM}$ and $\mathrm{CL}$. KK contributed to the data analysis and interpretation. All members of the team were active in preparing and revising the manuscript.

Competing interests AL is a Physical Activity Clinical Champion for Public Health England

Ethics approval Sheffield Hallam University, Faculty of Health \& Wellbeing Ethics Committee.

Provenance and peer review Not commissioned; internally peer reviewed.

Open Access This is an Open Access article distributed in accordance with the Creative Commons Attribution Non Commercial (CC BY-NC 4.0) license, which permits others to distribute, remix, adapt, build upon this work noncommercially, and license their derivative works on different terms, provided the original work is properly cited and the use is non-commercial. See: http:// creativecommons.org/licenses/by-nc/4.0/

(c) Article author(s) (or their employer(s) unless otherwise stated in the text of the article) 2017. All rights reserved. No commercial use is permitted unless otherwise expressly granted.

\section{REFERENCES}

1. Public Health England. Everybody active, every day: an evidencebased approach to physical activity. https://www.gov.uk/ government/publications/everybody-active-every-day-a-frameworkto-embed-physical-activity-into-daily-life (accessed 31 Aug 2017).

2. Lee IM, Shiroma EJ, Lobelo F, et al. Effect of physical inactivity on major non-communicable diseases worldwide: an analysis of burden of disease and life expectancy. Lancet 2012;380:219-29.

3. Ding D, Lawson KD, Kolbe-Alexander TL, et al. The economic burden of physical inactivity: a global analysis of major noncommunicable diseases. Lancet 2016;388:1311-24.

4. Sari N. Physical inactivity and its impact on healthcare utilization. Health Econ 2009;18:885-901.

5. Department of Health. Start active, stay active: a report on physical activity from the four home countries' chief medical officers London: Crowne, 2011.

6. World Health Organisation. Global Strategy Global Strategy on Diet, Physical Activity and Health. http://www.who.int/dietphysicalactivity/ strategy/eb11344/strategy_english_web.pdf?ua=1 (accessed 15 Apr 2017).

7. International society for physical activity and health. The Bangkok declaration on physical activity for global health and sustainable development. $2016 \mathrm{https} / / /$ static1.squarespace.com/static/559a3ff1 e4b0b0193b9d9862/t/5843cdfbe3df28eae5f43c10/1480838663699/ BKK_Declaration+FINAL+Dec2.pdf (accessed 15 Apr 17)

8. Sallis JF, Cervero RB, Ascher W, et al. An ecological approach to creating active living communities. Annu Rev Public Health 2006;27:297-322.

9. Global advocacy for physical activity (GAPA) the advocacy council of the International society for physical activity and health (ISPAH). 
NCD prevention: investments that work for physical activity. 2011 www.globalpa.org.uk/investmentsthatwork (accessed 15 Apr 2017).

10. Pears S, Morton K, Bijker M, et al. Development and feasibility study of very brief interventions for physical activity in primary care. $B M C$ Public Health 2015;15:333.

11. GC V, Wilson EC, Suhrcke M, et al. Are brief interventions to increase physical activity cost-effective? A systematic review. $\mathrm{Br} J$ Sports Med 2016;50:408.

12. National Institute for Health and Care Excellence. Behaviour change: Individual approaches. London: National Institute for Health and Care Excellence, 2014.

13. Health Education England. Making every contact count guidance documents. 2016 http://www.makingeverycontactcount.co.uk/ evidence/guidance/ (accessed 15 Apr 2017)

14. Health and Care Professions Council. Statistics - current number of registrants. 2016 http://www.hcpc-uk.co.uk/aboutregistration/ theregister/stats/ (accessed 15 Apr 2017).

15. Health and Social Care Information Centre. Hospital Outpatient Activity 2015-16. http://www.content.digital.nhs.uk/catalogue/ PUB22596 (accessed 15 Apr 2017).

16. McPhail S. Multi-morbidity, obesity and quality of life among physically inactive Australians accessing physiotherapy clinics for musculoskeletal disorders. Physiotherapy 2015;101:e986-7.

17. Barrett EM, Darker CD, Hussey J. Promotion of physical activity in primary care: knowledge and practice of general practitioners and physiotherapists. J Public Health 2013;21:63-9.

18. Aweto HA, Oligbo CN, Fapojuwo OA, et al. Knowledge, attitude and practice of physiotherapists towards promotion of physically active lifestyles in patient management. BMC Health Serv Res 2013;13:21.

19. Radež $P$, Šcepanovic $D$, Juričan $A B$. The physiotherapy and physical activity components within the antenatal classes in Slovenia. Physiotherapy 2015;101:e100-1.

20. Lau C, Chitussi D, Elliot S, et al. Facilitating community-based exercise for people with stroke: a cross-sectional e-survey of physical therapy practice and perceived needs. Phys Ther 2016;96:e1323.

21. Shirley $D$, van der Ploeg HP, Bauman AE. Physical activity promotion in the physical therapy setting: perspectives from practitioners and students. Phys Ther 2010;90:1311-22.

22. O'Donoghue G, Cunningham C, Murphy F, et al. Assessment and management of risk factors for the prevention of lifestyle-related disease: a cross-sectional survey of current activities, barriers and perceived training needs of primary care physiotherapists in the Republic of Ireland. Physiotherapy 2014;100:116-22.

23. Lowe A, Gee M, McLean S, et al. Physical activity promotion in physiotherapy practice: a systematic scoping review of a decade of literature. Br J Sports Med 2016:bjsports-2016-096735 (Epub ahead of print: 21 Dec 2016).

24. Department of Health. Start active, stay active: infographics on physical activity. https://www.gov.uk/government/uploads/system/ uploads/attachment_data/file/541233/Physical_activity_infographic. PDF (accessed 15 Apr 2017)

25. Bates S, Kipps C. An anonymous online survey of the views and attitudes of medical students and junior doctors towards physical activity teaching and promotion. Br J Sports Med 2013;47:e3.46-e3.

26. Weiler $\mathrm{R}$, Chew $\mathrm{S}$, Coombs $\mathrm{N}$, et al. Physical activity education in the undergraduate curricula of all UK medical schools: are tomorrow's doctors equipped to follow clinical guidelines? $\mathrm{Br} J$ Sports Med 2012;46:1024-6.

27. Dunlop M, Murray AD. Major limitations in knowledge of physical activity guidelines among UK medical students revealed: implications for the undergraduate medical curriculum. $\mathrm{Br} \mathrm{J}$ Sports Med 2013;47:718-20.

28. NHS England, Care Quality Commission, Health Education England, Monitor, Public Health England, Trust Development Authority. NHS five year forward view. London: NHS England, 2014.

29. National Institute for Health and Care Excellence. Quality Standard 84: Physical activity: for NHS staff, patients and carers. https://www. nice.org.uk/guidance/qs84 (accessed 15 May 2017).

30. NHS England NHS staff health \& wellbeing: CQUIN Supplementary guidance. https://www.england.nhs.uk/wp-content/ uploads/2016/03/HWB-CQUIN-Guidance.pdf (accessed 15 May 2017).

31. Lobelo F, de Quevedo IG. The evidence in support of physicians and health care providers as physical activity role models. Am J Lifestyle Med 2016;10:36-52.

32. Mouton A, Mugnier B, Demoulin C, et al. Physical therapists' knowledge, attitudes, and beliefs about physical activity: a prerequisite to their role in physical activity promotion? J Phys Ther Edu 2014;28:120-7.

33. Milton K, Bull FC, Bauman A. Reliability and validity testing of a single-item physical activity measure. Br J Sports Med 2011;45:203-8.

34. von Elm E, Altman DG, Egger M, et al. The Strengthening the Reporting of Observational Studies in Epidemiology (STROBE) statement: guidelines for reporting observational studies. J Clin Epidemiol 2008;61:344-9.

35. Walkeden S, Walker KM. Perceptions of physiotherapists about their role in health promotion at an acute hospital: a qualitative study. Physiotherapy 2015;101:226-31.

36. Hébert ET, Caughy MO, Shuval K. Primary care providers' perceptions of physical activity counselling in a clinical setting: a systematic review. Br J Sports Med 2012;46:625-31.

37. NHS England.. The General Practice Physical Activity Questionnaire (GPPAQ) a screening tool to assess adult physical activity levels, within primary care. https://www.gov.uk/government/uploads/ system/uploads/attachment_data/file/192453/GPPAQ_-_guidance. pdf (accessed 15 May 2017).

38. National institute for health and care excellence. Physical activity: brief advice for adults in primary care. https://www.nice.org.uk/ guidance/ph44 (accessed 15 May 2017).

39. Bull FC, Milton KE, Karen M. A process evaluation of a "physical activity pathway" in the primary care setting. BMC Public Health 2010;10:463.

40. Aveyard P, Lewis A, Tearne S, et al. Screening and brief intervention for obesity in primary care: a parallel, two-arm, randomised trial. The Lancet 2016;388:2492-500.

41. Vidrine JI, Shete S, Cao Y, et al. Ask-Advise-Connect: a new approach to smoking treatment delivery in health care settings. JAMA Intern Med 2013;173:458-64.

42. Reid $\mathrm{H}$, Milton $\mathrm{K}$, Bownes $\mathrm{G}$, et al. Making physical activity evidence accessible: are these infographics the answer? Br J Sports Med 2017;51:764-6.

43. Prince SA, Adamo KB, Hamel ME, et al. A comparison of direct versus self-report measures for assessing physical activity in adults: a systematic review. Int J Behav Nutr Phys Act 2008;5:56.

44. Neil-Sztramko SE, Ghayyur A, Edwards J, et al. Physical activity levels of physiotherapists across practice settings: a cross sectional comparison using self-report questionnaire and accelerometer measures. Physiother Can 2017;69:1-9 